\title{
Linnda R. Caporael, James R. Griesemer, and William C. Wimsatt (eds): Developing Scaffolds in Evolution, Culture, and Cognition*
}

MIT Press, Cambridge, MA, 2013, 426 + xiv pp, US\$ 60.00/£ 41.95 (Hb), ISBN: 9780262019552

\author{
Joseph D. Martin
}

To understand what this volume is, we must begin with what it isn't. That story starts a century ago when physics was king. Philosophers, economists, and sociologists appropriated tools forged for classical mechanics and thermodynamics as they tried to develop rigorous scientific explanations for human social phenomena (see Porter 1995). Through the century after the heyday of the Vienna Circle, the life sciences began to command the greatest share of scientific funding, prestige, and influence and we began asking whether evolutionary biology might offer a more apt basis for sound scientific insight into human culture and cognition. As important a transition as this was, it preserved many methodological assumptions that originated in the physical sciences and so early attempts to explain cognition and culture in biological terms proceeded from the same type of reductionist thinking that logical positivism epitomized. These are the foil for this collection, which considers evolution, culture, and cognition as mutually supporting processes rather than trying to understand the second two as straightforward consequences of the first.

The common theme among the approaches against which this volume reacts is a tendency to cast cultural or cognitive phenomena as extensions of more basic biological processes. Memetics defines an individuated unit, the meme, to act as a selfish self-replicator that can be modeled like gene selection (Dawkins 1976). Evolutionary Psychology postulates that conditions in early human history offer selective explanations today's psychological and cultural landscape (Barkow et al. 1992). These approaches reflect a longstanding and widely adopted presumption that culture is explicable in terms of basic biological laws. Caporael, Griesemer, and Wimsatt set this volume in direct opposition to what they call "the gene's eye view" of biology and human societies, eschewing the reductive assumptions that often accompany gene selectionism and population genetics. Instead, their positive program asks us to consider "scaffolding" as an overarching framework that can be adapted to evolutionary, developmental, cognitive, social, and cultural change at all scales.

What is scaffolding in this context? When understood as identifying something in the world, scaffolds are the supporting structures and processes that help individuals or systems acquire new capacities. Examples might include a cell's role in supporting virus production, the caregiver interactions that help children develop language proficiency, and the training regimes and socialization processes that allow sports teams to function as a cooperative unit. Speaking more broadly, scaffolding is a theoretical tool that complements neo-Darwinism by giving a larger role to development while identifying and filling in the gaps where population genetics has little to say. Scaffolding structures and scaffolding processes-the concept's entity/process duality is criticalsupport the developmental trajectories experienced by adaptive systems at all scales. Scaffolds are often rendered obscure once they complete their supporting roles and fall away. Like the wooden

\footnotetext{
${ }^{*}$ The final publication is available at Springer via http://dx.doi.org/10.1007/s10441-014-9230-z.
} 
scaffolds that support an arch while it is being assembled or allow a worker to repair the stonework on a crumbling cathedral, biological, cognitive, and cultural scaffolds are critical to the acquisition, transmission, and maintenance of developmental capacities and yet the details of their operation are difficult to infer from the finished product. Whether scaffolding is a metaphor, a heuristic, or a full-fledged theoretical program bears further discussion, but it will be useful first to consider the vocabulary through which the editors evolve it and how the contributors to this volume deploy it.

Each editor contributes a piece to the book's first section. These three essays develop the suite of conceptual tools that the editors aim to unite within the scaffolding approach. Griesemer begins by describing the reproduce perspective, defining reproduction as a process in which material overlap between an entity and its offspring confers developmental capacities to the offspring. This definition encompasses obvious examples like the transfer of genetic material during gamete fusion, but it is also interesting for highlighting the differences between biological reproduction, for which material overlap is central, and cultural transmission, for which it is not. Caporael introduces the concepts of repeated assembly and core configurations. At the most general level, repeated assemblies are recurrent interactions between entities and their environments that play some role themselves in their own recurrence. Examples could include everything from protein folding to the annual meeting of a professional society. The particular examples Caporael highlights are core configurations, human groups of roughly similar size that have been associated with particular types of tasks across cultures in human history. Finally, W. Wimsatt introduces entrenchment: the notion that the more richly connected a feature of a dynamic system is to downstream consequences for the system, the more that feature will resist perturbation. A gene with a great many developmental consequences will be less prone to change through selective processes, just as deeply rooted cultural practices that shape the ordering and function of an institution often prove difficult to dislodge.

Two similarities among this suite of concepts make up the conceptual core of the approach this volume describes and illustrates. First, these concepts are scale insensitive. They can be applied equally well at many levels of organization and so avoid privileging a particular scale of analysis. The reproducer perspective can capture DNA-based genetic transfer, but it also describes the process by which a new company forms when a subset of employees breaks away from and old one, as happened in the late 1950s when technical staff began an exodus from Shockley Semiconductor Laboratory, precipitating the growth of Silicon Valley (Riordan and Hoddeson 1997). In the same manner, repeated assemblies and entrenchment, as seen in the examples offered above, can be found with equal ease on the molecular scale and the institutional scale.

Second, these concepts treat boundaries of all kinds as conventional. They are not committed to hard and fast distinctions among entities, between entities and their environments, or between entities and processes. Much to the contrary, the framework that emerges here insists that the precise location of these boundaries depends upon an interpretive frame of reference. From the reproducer perspective, for instance, we see material overlap from individual to gamete and gamete to zygote, but it does not imbue any one of these stages with special ontological status. Rather, Griesemer suggests that how biologists choose to trace particular features of this process is critical for developing an entity's provisional definitional boundaries. Likewise, repeated assembly and 
entrenchment focus our attention on processes, in which the exact parameters for individuating the entities participating in those processes are secondary to how one constructs them.

Scale insensitivity and the conventionality of boundaries are the leitmotifs for the remainder of the volume, which consists of articles that either apply one or more of the editors' concepts directly or use an approach that is consonant with them. Flexibility across scope and scale is highlighted in Section II, which examines how constraints provided by the physical properties of critical molecules scaffolds the development of embryonic body plans (Newman), how the form and structure of communication media scaffold human cognition (Evans), and how model building can be construed as scaffolding for scientific understanding (Schank et al.). Although they consider scales from molecules to human communities and temporal spans from deep evolutionary time to the timeline of an experiment, these three studies use scaffolding in the same substantive way, highlighting developmentally crucial features of ostensibly radically different processes that would otherwise get short shrift. By grouping these pieces, the editors illustrate how scale-invariant concepts help hedge against charges of pernicious oversimplification that bedevil gene-level approaches to culture and cognition.

Section III brings the question of boundaries to the fore. Lyon shows how the evolution of human cognition is intimately intertwined with external stressors; Theiner argues that the group should be considered the relevant cognitive agent in certain contexts; Heintz describes a rich process of codetermination between cognition and culture that calls into question any strict boundary between the two. Read against the theoretical background provided in Section I, these essays crystalize the point that drawing boundaries or defining relationships, such as scaffolding relationships, is a theory-laden act and should therefore be explicitly articulated with reference to a set of interpretive goals. Having units of analysis is important, but defining them is in itself an act of interpretation, especially when dealing with the complexities of culture.

As should be evident by now, the editors consciously avoided grouping the contributions into traditional subject or disciplinary categories such as evolution, cognition, and culture, or biology, philosophy, and social science, instead hoping to highlight common themes across papers written with different topics and methodological aims. This strategy, which exposed novel consonances between the papers in Sections II and III, begins to strain in Sections IV and V, which do not exhibit the same thematic consistency. In Section IV, Allen discusses how symbols, entrenched as parts of various communication systems, scaffold cognitive development. Martínez brings a similar approach to artifacts and diagrams. Gerson, in a piece that might have been more effective earlier in the volume, takes a more abstract stance by adopting an institution-level perspective on the question of how we should define culture and cultural change and summarizing the difficulties that arise from straightforward analogies between evolutionary and cultural change. The goal of this section is to show reciprocal relationships between processes at different scales, but few such connections are drawn explicitly in the papers themselves leaving it to the reader to make that leap.

Section V is entitled "Reproduction and Development," a category general enough that almost any of the volume's contributions might have fit within it. The four papers here, each interesting and insightful in its own right, fit together less by being applications of the concepts articulated earlier in the volume than they do by being independent expressions of similar ideas. Murman argues that 
evolutionary approaches to economic development can benefit from considering how institutional scaffolding helps individual firms acquire the capacities they need to survive. Contributions from Tavory et al. and Li make no mention of scaffolding, but nonetheless resonate with the volume's objectives. The former develops a developmental system theory approach to cultural evolution, arguing that to understand how cultural phenomena perpetuate themselves we need to take the systems in which they are expressed as our unit of analysis, rather than looking for a potent causal agent at some lower level of complexity. The latter's discussion of brain plasticity provides a basis for discussing how large-scale cultural processes exert influence on human development, and so should be taken seriously, as biological processes. Finally, B. Wimsatt provides the most direct and reflexive application of the volume's core concepts by considering how different varieties of scaffolding shape scientific career trajectories at different stages.

In the final analysis, the advantages of a novel organizational scheme outweigh the lack of crisp thematic overlap among the papers in the later sections. The volume is premised on breaking out of traditional categories. If the groupings offered in their place fail to crystalize they at least encourage the reader to seek out other connections between the papers. The range of topics and methodologies evident here mean that it can be broken down in many different ways. This makes the volume challenging and sometimes chaotic, but is in keeping with a methodological insistence on the conventional nature of boundaries.

Given the diversity of approaches and topics in this volume it is understandable that "scaffolding," the orienting concept, is applied differently throughout. The editors view it as a theoretical program, albeit an inchoate one. For some contributors it is a critical theoretical concept, elaborated through its application. For others it is a heuristic or a metaphor. Some ambiguities persist about what should count as scaffolding and what should not. As a result, readers might find that a clear theoretical program fails to emerge, or does so only weakly. This is a consequence of both the breadth of the scaffolding concept and the multidisciplinary nature of its deployment in this volume. But it would be premature to assess this volume on the success or failure of a coherent theoretical structure. Instead, we should ask what scaffolding can accomplish.

Whether the approach developed here counts as a full-fledged theoretical program or not, it does develop a vocabulary for discussing biological phenomena at all scales of complexity. That vocabulary rests on some novel methodological tenets. Scaffolding, entrenchment, repeated assembly, reproducers, and core configurations encourage the examination of biological, cognitive, and cultural processes across scales and with sensitivity to how drawing conceptual boundaries influences theoretical judgments. On this view, it is not so critical that scaffolding really exists either as a thing in the world or as a consistently applied theoretical program, but rather that it advances fruitful conceptual and methodological tools. The range of contributions in the volume testifies to the potential of the scaffolding approach to be fruitful indeed. Consider this book in its own language: it remains to be seen if the theoretical program the editors foresee will become entrenched, but even if it does not, at the very least it is a scaffold. This book frames provocative question and identifies new investigative directions for theoretical biology. Even if the program described here turns out to be a temporary platform, it is at least a place where future researchers might stand while building something permanent. 


\section{Reference List}

Barkow, J. H., L. Cosmides, and J. Tooby (eds.) (1992) The adapted mind: Evolutionary Psychology and the generation of culture. New York: Oxford University Press.

Dawkins, R. (1976) The selfish gene. Oxford University Press, Oxford, UK.

Porter, T (1995) Trust in numbers. Princeton University Press, Princeton.

Riordan, M. and L. Hoddeson (1997) Crystal fire: The birth of the information age. W. W. Norton \& Co., New York. 